\title{
Epidemiologia da Vertigem na Urgência Hospitalar
}

\section{Epidemiology of Vertigo on Hospital Emergency}

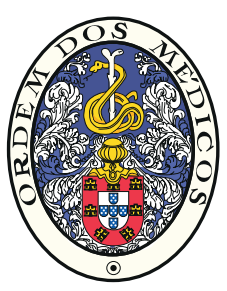

Luís Roque REIS $\triangle^{1,2}$, Rita LAMEIRAS ${ }^{1}$, Pedro CAVILHAS ${ }^{1}$, Pedro ESCADA ${ }^{1,2}$

Acta Med Port 2016 May;29(5):326-331 - http://dx.doi.org/10.20344/amp.6571

RESUMO

Introdução: A vertigem é um dos motivos mais frequentes na procura de atendimento médico especializado na urgência. O objetivo deste estudo foi avaliar o peso real que as alterações do equilíbrio têm na urgência de Otorrinolaringologia, as suas características epidemiológicas e uma eventual sazonalidade por estações do ano. Usou-se uma amostra de doentes que utilizou o serviço de urgência desta especialidade de um hospital central universitário de Lisboa durante um período de quatro anos.

Material e Métodos: O projeto foi concebido como um estudo epidemiológico descritivo de tipo populacional, retrospectivo, no período de 2010 a 2013. Incluiu uma população total de 40173 atendimentos de doentes que nesse período utilizou o serviço de urgência de Otorrinolaringologia. As variáveis estudadas incluíram a idade, género, número anual de casos (total e percentual), data da crise de vertigem, distribuição sazonal por estações do ano e proporção anual de casos internados.

Resultados: Um total de 4347 doentes (10,8\%) procurou atendimento médico devido a vertigem ou alterações do equilíbrio ao longo dos quatro anos do estudo. Verificou-se um aumento anual do número de casos entre 7,6\% (em 2010) a 17\% (em 2013). As mulheres foram mais frequentemente afetadas $(68,3 \%)$ e as crises ocorreram mais frequentemente em indivíduos entre os 60 e 79 anos de idade (40\%). Os casos distribuíram-se de forma heterogénea entre as estações do ano, havendo mais episódios de vertigem no verão e no outono e com uma tendência crescente entre 2010 e 2013. O número de internamentos aumentou anualmente nesse período.

Discussão: A epidemiologia da vertigem e dos distúrbios vestibulares específicos é ainda um campo a estudar, pois pode ter utilidade para a tomada de decisões clínicas e para o planeamento dos cuidados de saúde.

Conclusão: $O$ estudo revelou que os casos de vertigem na urgência aumentaram anualmente e são mais frequentes no sexo feminino, na população idosa e no verão e outono.

Palavras-chave: Portugal; Serviço de Urgência Hospitalar; Vertigem/epidemiologia.

\section{ABSTRACT}

Introduction: Vertigo is one of the most frequent motives in seeking specialized medical care in an emergency. The aim of this study was to evaluate the relevance of balance disorders in the Otorhinolaryngology urgency, the epidemiological characteristics and the possible seasonality. It was used a sample of patients who were assisted in a health care emergency department of this specialty, of a universitary hospital and during a period of four years.

Material and Methods: The project was conceived as a descriptive epidemiological study of population type, retrospective, during the period from 2010 to 2013. A total population of 40,173 attendances of patients during this period used the emergency eepartment of Otolaryngology. The parameters studied included age, gender, annual number of cases (total and percentage), date of crisis of vertigo, seasonal distribution by seasons and annual proportion of hospitalized cases.

Results: A total of 4,347 patients (10.8\%) sought medical attention due to dizziness/balance disorders over the four years of the study. There has been an annual increase in the number of cases between $7.6 \%$ (in 2010) and 17\% (in 2013). Women were more often affected (68.3\%) and crises occurred more often in individuals between 60 and 79 years of age (40\%). The cases were distributed heterogeneous between the seasons, having more episodes of dizziness in summer and autumn and with an increasing trend between 2010 and 2013. The number of hospitalizations has increased annually over this period.

Discussion: The epidemiology of vertigo and vestibular disorders is still a specific field to study, because it may be useful for clinical decision-making and health care planning.

Conclusion: The study revealed that cases of vertigo in urgency increased annually and are more frequent in women, in elderly population and in Summer and Autumn.

Keywords: Emergency Service, Hospital; Portugal; Vertigo/epidemiology.

\section{INTRODUÇÃO}

A vertigem é um sintoma subjetivo e difícil de definir, que pode ter diversas causas e gravidade de ligeira até muito grave. Embora muitos médicos e doentes usem os termos vertigem e tonturas indistintamente, o diagnóstico diferencial destas situações é determinante, devendo-se desde logo diferenciar a vertigem, que tem uma causa vestibular (periférica ou central), da tontura, de causa não vestibular. A Comissão de Audição e Equilíbrio da Academia Americana de Otorrinolaringologia e Cirurgia Cérvico-Facial definiu vertigem como uma "sensação de movimento quando nenhum movimento está a ocorrer em relação à gravidade da terra". 1

A existência de dados epidemiológicos disponíveis sobre a vertigem e sobre as síndromes vestibulares permitiria ajudar os clínicos a tomarem melhores decisões, baseadas na evidência. Contudo, apesar das tonturas e vertigens serem referidas como uma das dez principais causas de encaminhamento para o Serviço de Urgência ${ }^{2}$ e um motivo frequente de observação na Urgência de Otorrinolaringologia (ORL), a epidemiologia da vertigem apontada para

1. Serviço de Otorrinolaringologia. Hospital Egas Moniz. Centro Hospitalar de Lisboa Ocidental. Lisboa. Portugal.

2. Departamento de Otorrinolaringologia. Faculdade de Ciências Médicas. Lisboa. Portugal.

$\bowtie$ Autor correspondente: Luís Reis. roque-reis@sapo.pt

Recebido: 03 de maio de 2015 - Aceite: 16 de dezembro de 2015 | Copyright @ Ordem dos Médicos 2016 
a população geral é variável: os dois maiores estudos, de Mendel et $\mathrm{al}^{3}$ e Neuhauser et al, ${ }^{4}$ indicam valores entre $4,8 \%$ e $10,5 \%$ para a prevalência da vertigem de causa vestibular. Por sua vez, Hain ${ }^{5}$ estima que $15 \%$ da população tem alterações do equilíbrio de qualquer causa (vestibular e não vestibular), o que sugere que um terço a dois terços de todos os doentes com alterações do equilíbrio terão problemas no ouvido interno. Estimou-se ainda que em países onde os cuidados de saúde são menos centrados no serviço de urgência do que em Portugal, os doentes em seguimento nos cuidados de saúde por vertigem num determinado momento, tiveram um número de observações médicas nos serviços de urgência de 0,4 \pm 0,9 observações e $2 \pm 5,4$ dias de internamento hospitalar nos três meses precedentes, por causa da vertigem. ${ }^{6}$

O objetivo principal deste estudo é o de identificar a importância que as alterações do equilíbrio têm num serviço de urgência de otorrinolaringologia. Este é provavelmente o primeiro artigo a abordar esta temática em Portugal. O estudo, retrospectivo, descreve os aspetos epidemiológicos da vertigem nos pacientes que recorreram ao Serviço de Urgência (SU) de Otorrinolaringologia do Hospital de Egas Moniz. Outro aspeto original deste trabalho é o facto de procurar esclarecer se a vertigem tem sazonalidade. São analisadas as seguintes variáveis: distribuição anual em relação à idade, género, estação do ano, distribuição anual por estações, número anual de casos (total e percentual) e número anual de casos de internamento. Será verificado se existem alterações estatisticamente significativas, ao longo do período do estudo, na distribuição anual, por estação do ano e no número de casos de internamento.

\section{MATERIAL E MÉTODOS}

Realizou-se uma pesquisa na base de dados do Serviço de Urgência (SU) de ORL, através do Serviço de Sistemas e Tecnologias de Informação (SSTI) do Centro Hospitalar de Lisboa Ocidental (CHLO), EPE. Uma vez que são mais do que vinte os médicos que observam os doentes e codificam os diagnósticos de urgência no sistema informático, há naturalmente alguma variabilidade de critérios e diagnósticos. Por essa razão selecionaram-se para o estudo todos

Tabela 1 - Diagnósticos e códigos de diagnóstico dos doentes selecionados para o estudo

\begin{tabular}{lc}
\hline Diagnóstico & Código ICD* \\
\hline Vertigem de origem central & 386.2 \\
Vertigem epidémica & 078.01 \\
Vertigem periférica SOE & 386.10 \\
Vertigem paroxística benigna posicional & 386.11 \\
Vertigem periférica NCOP & 386.19 \\
Vertigem & 438.85 \\
Tonturas e vertigens & 780.4 \\
\hline *International Classification of Diseases, 9th revision, Clinical Modification (ICD-9-CM) \\
SOE: sem outra especificação; NCOP: não classificável em outra parte.
\end{tabular}

os códigos associados ao diagnóstico de vertigem (Tabela 1): este estudo visa a avaliação global da distribuição das alterações do equilíbrio e vertigem, e não especificamente por patologia. Os sintomas "vertigem" e "tontura" são combinados na variável "tonturas e vertigens" do código 780.4.

Foram considerados todos os doentes consultados no SU de ORL do CHLO de 1 de Janeiro de 2010 a 31 de Dezembro de 2013 (quatro anos). Este SU funciona como uma urgência aberta (sem triagem) que tem como área de influência cinco freguesias do concelho de Lisboa (São Francisco Xavier, Santa Maria de Belém, Alcântara, Santo Condestável e Ajuda) e os concelhos de Oeiras e Cascais, prestando cuidados de saúde a uma população de 439944 habitantes (censos de 2011). É uma região do litoral oeste da Portugal, com uma população predominantemente caucasiana, em que o acesso aos cuidados de saúde é universal mas sujeita a pagamento (taxa moderadora) dos cuidados médicos.

O período de tempo total considerado para o estudo foi alargado, de quatro anos, para aumentar a precisão estatística e permitir uma análise epidemiológica ao longo do tempo. Foi estudada a distribuição: quer dos casos (diagnóstico de vertigem); quer do número total de episódios de urgência, nos referidos períodos de tempo (Tabela 2).

Foram ainda recolhidos os seguintes dados do SSTI: nome, género, idade, data da vinda ao SU considerada como a data da crise de vertigem e doentes que foram internados com esta patologia pelo SU. Os dados foram organizados e estratificados em escalões etários por décadas (intervalos de dez anos), por género e por estações do ano agrupadas (verão-outono e inverno-primavera).

Introduzimos os dados recolhidos na folha de cálculo Excel $^{\circledR}$, sendo a análise estatística realizada utilizando o Statistical Package for the Social Sciences ${ }^{\circledR}$ (SPSS) versão 21.0 para Windows ${ }^{\circledR}$. Numa primeira fase testámos os pressupostos para a utilização de testes paramétricos, para orientar a escolha dos testes mais apropriados. As análises realizadas e os testes que foram utilizados estão indicados na Tabela 3.

O presente estudo foi elaborado segundo a norma da DGS 015/2013 de 03/10/2013 de consentimento informado e em particular do referido nas alíneas $G$ ) e R).

\section{RESULTADOS}

Verificou-se um aumento do número absoluto de casos de vertigem entre os anos de 2010 e 2013, enquanto que concomitantemente se verificou um decréscimo ligeiro do número total de urgências. Em consequência a razão entre o número de casos de vertigem por ano e o número total de urgências por ano traduziu um aumento percentual dos casos no período referido entre $7,6 \%$ a $17 \%$ (Tabela 4 ).

A Tabela 5 apresenta a distribuição dos episódios de vertigens entre género, escalões etários, estações do ano, estações do ano agrupadas (inverno e primavera versus verão e outono). Existem diferenças estatisticamente significativas dos episódios de vertigem entre género $\chi^{2}[1]$ $=579,381 ; p<0,001)$, escalões etários $\left(\chi^{2}[9]=2\right.$ 033,370; 
Tabela 2 - Períodos de tempo do estudo

Período de tempo

Tempo total do estudo (1 de janeiro de 2010 a 31 de dezembro de 2013 - 4 anos)

Anual (de cada ano: 2010, 2011, 2012 e 2013)

Sazonal (por estações do ano, em cada um dos anos 2010 a 2013)

Verão: de 21 de junho a 22 de setembro

Outono: de 23 de setembro a 20 de setembro

Inverno: de 21 de dezembro a 20 de março

Primavera: de 21 de março a 20 de junho

Tabela 3 - Análises estatísticas realizadas e testes utilizados

\begin{tabular}{cccc}
\hline Análise & Variável & Teste \\
\hline
\end{tabular}

Homogeneidade da distribuição

Associação com estações do ano

Ano

$\mathrm{N}^{\circ}$ de internamentos, \% de casos no total de urgências,

Associação com ano $\mathrm{n}^{\circ}$ total de urgências, percentagem de internamentos no total de casos
Qui-quadrado de ajustamento

Qui-quadrado de associação

Coeficiente de correlação de Pearson

Tabela 4 - Distribuição do número de casos de vertigem (Nvert) e o número total de urgências por ano (Ntotal).

\begin{tabular}{cccccccccccc}
\hline & \multicolumn{2}{c}{2010} & \multicolumn{4}{c}{2011} & \multicolumn{3}{c}{2012} & \multicolumn{3}{c}{2013} \\
Ntotal & Nvert & $\%$ & Ntotal & Nvert & $\%$ & Ntotal & Nvert & $\%$ & Ntotal & Nvert & $\%$ \\
\hline 11256 & 860 & 7,6 & 11285 & 974 & 8,6 & 9127 & 1064 & 11,7 & 8505 & 1449 & 17,0 \\
\hline
\end{tabular}

$p<0,001)$, estações do ano $\left(\chi^{2}[3]=14,197 ; p=0,003\right)$, estações do ano agrupadas $\left(\chi^{2}[1]=7,536 ; p=0,006\right) \mathrm{e}$ entre os anos $\left(\chi^{2}[3]=180,235 ; p<0,001\right)$. Os resultados mostram que há mais mulheres com episódios de vertigem (68.3\%), mais episódios de vertigem entre os 70 e os 79 anos $(20,8 \%)$ e em particular na faixa etária dos 60 aos 79 anos (40\%), mais episódios de vertigem no verão e no outono $(52,1 \%)$ e que os episódios de vertigem têm mostrado uma tendência crescente desde 2010 (7,6\%) a 2013 (17\%).

A Tabela 6 apresenta os resultados da associação entre a sazonalidade e o ano do estudo. Foi encontrada essa associação $\left(\chi^{2}[9]=19,780 ; p=0,019\right)$, mas apesar da estação com registo de mais casos de vertigem ser o verão ou o outono na análise do período total do estudo, foi o verão a estação com mais casos em $2010(27,1 \%)$ e 2013 (28\%), o inverno em 2011 (26,1\%) e o outono em 2012 (31\%).

Os resultados da associação entre o ano e o número de internamentos, percentagem de casos no total das urgências, número total de urgências e percentagem de internamentos no total de casos são apresentados na Tabela 7. O ano correlacionou-se de forma positiva, forte e estatisticamente significativa com o número de internamentos $(r=0,98 ; p=0,019)$ e com a percentagem de casos no total das urgências $(r=0,95 ; p=0,046)$. Os resultados mostraram que os internamentos (37 casos) e a percentagem de casos no total das urgências aumentaram de forma es- tatisticamente significativa entre 2010 (sete casos) e 2013 (12 casos), mas que não houve crescimento significativo na percentagem de internamentos no total de casos.

\section{DISCUSSÃO}

Neste artigo, os autores pretendem dar uma visão sobre a epidemiologia da vertigem como causa de atendimento num serviço de urgência de otorrinolaringologia em Portugal. Um total de 4347 doentes (10,8\%) foi observado por vertigem/alterações do equilíbrio ao longo de 4 anos. Verificou-se um aumento anual do número de casos, de $7,6 \%$ para $17 \%$ dos doentes observados no mesmo serviço de urgência. As mulheres e os indivíduos entre os 60 e 79 anos de idade foram mais frequentemente afetados. Registaram-se mais episódios de vertigem no verão e no outono, com uma tendência crescente entre 2010 e 2013. O número de internamentos aumentou anualmente, mas não quando ajustado ao número anual total de casos.

Os resultados do estudo não permitem documentar a incidência e prevalência da doença na população em geral. Só é possível identificar a proporção dos casos de vertigem no conjunto dos atendimentos na urgência de ORL. Contudo podemos assinalar o número elevado e crescente de casos, que representaram quase um quinto dos motivos observação na urgência de ORL. Podemos conjeturar, pela análise destes resultados, que a vertigem tem uma 
Tabela 5 - Distribuição por género, escalão etário, estação do ano, estação do ano agrupada e ano.

\begin{tabular}{|c|c|c|c|c|}
\hline & $\mathrm{N}$ & $\%$ & $\chi^{2}$ & $p$ \\
\hline Género & & & 579,381 & $<0,001$ \\
\hline Masculino & 1380 & 31,7 & & \\
\hline Feminino & 2967 & 68,3 & & \\
\hline Escalão etário & & & 2033,370 & $<0,001$ \\
\hline $0-9$ & 24 & 0,6 & & \\
\hline $10-19$ & 130 & 3,0 & & \\
\hline $20-29$ & 236 & 5,4 & & \\
\hline $30-39$ & 431 & 9,9 & & \\
\hline $40-49$ & 526 & 12,1 & & \\
\hline $50-59$ & 610 & 14,0 & & \\
\hline $60-69$ & 835 & 19,2 & & \\
\hline $70-79$ & 905 & 20,8 & & \\
\hline $80-89$ & 588 & 13,5 & & \\
\hline 90 ou mais anos & 62 & 1,4 & & \\
\hline Estação do ano & & & 14197 & 0,003 \\
\hline Primavera & 1011 & 23,3 & & \\
\hline Verão & 1149 & 26,4 & & \\
\hline Outono & 1147 & 26,4 & & \\
\hline Inverno & 1040 & 23,9 & & \\
\hline Estação do ano agrupada & & & 7536 & 0,006 \\
\hline Inverno e primavera & 2083 & 47,9 & & \\
\hline Verão e outono & 2264 & 52,1 & & \\
\hline Ano & & & 180235 & $<0,001$ \\
\hline 2010 & 860 & 19,8 & & \\
\hline 2011 & 974 & 22,4 & & \\
\hline 2012 & 1064 & 24,5 & & \\
\hline 2013 & 1449 & 33,3 & & \\
\hline
\end{tabular}

Tabela 6 - Distribuição por ano e estação do ano

\begin{tabular}{|c|c|c|c|c|c|c|c|c|c|c|}
\hline & \multicolumn{2}{|c|}{2010} & \multicolumn{2}{|c|}{2011} & \multicolumn{2}{|c|}{2012} & \multicolumn{2}{|c|}{2013} & \multirow[b]{2}{*}{$\chi^{2}$} & \multirow[b]{2}{*}{$p$} \\
\hline & $\mathrm{N}$ & $\%$ & $\mathrm{~N}$ & $\%$ & $\mathrm{~N}$ & $\%$ & $\mathrm{~N}$ & $\%$ & & \\
\hline Estação do ano & & & & & & & & & 19,780 & 0,019 \\
\hline Primavera & 199 & 23,1 & 237 & 24,3 & 230 & 21,6 & 345 & 23,8 & & \\
\hline Verão & 233 & 27,1 & 247 & 25,4 & 264 & 24,8 & 405 & 28,0 & & \\
\hline Outono & 225 & 26,2 & 236 & 24,2 & 330 & 31,0 & 356 & 24,6 & & \\
\hline Inverno & 203 & 23,6 & 254 & 26,1 & 240 & 22,6 & 343 & 23,7 & & \\
\hline
\end{tabular}

grande prevalência na população portuguesa, que o enveIhecimento da população contribui para este facto e que os doentes não conseguem obter um tratamento adequado através da articulação entre os cuidados de saúde primá- rios e a consulta de otorrinolaringologia (e daí que o doente recorra diretamente ao serviço de urgência).

Segundo Burt e Schappert ${ }^{7}$ as alterações do equilíbrio têm aumentado drasticamente ao longo do tempo e estão 
Tabela 7 - Associação entre ano e número de internamentos, percentagem de casos no total das urgências, número total de urgências e percentagem de internamentos no total de casos

\begin{tabular}{lc}
\hline & Ano \\
\hline Internamentos & $0,98^{*}$ \\
Percentagem de casos no total das urgências & $0,95^{*}$ \\
Total de urgências & $-0,93$ \\
Percentagem de internamentos no total de casos & 0,47 \\
\hline
\end{tabular}

Resultados expressos no coeficiente de correlação de Pearson; * $p \leq 0,05$

entre os motivos mais comuns de recurso ao SU. Têm um impacto substancial e crescente nos SU dos EUA e multiplicam-se ${ }^{8}$ mas as razões permanecem pouco claras: para Kerber et al ${ }^{8}$ é possível que este aspeto se deva ao envelhecimento da população mas também ao facto dos médicos dos cuidados de saúde primários cada vez mais instruírem os doentes com alterações do equilíbrio a recorrerem ao SU.

O nosso estudo é concordante com outros estudos existentes na literatura médica, que registam uma prevalência da vertigem no sexo feminino dupla da do sexo masculino, ou aproximadamente..$^{8-10} \mathrm{~A}$ maior prevalência da vertigem nos idosos que registámos no nosso estudo é concordante com os dados gerais da literatura, traduzindo as alterações vasculares e degenerativas do sistema vestibular que são mais prováveis de ocorrer em pessoas mais idosas. ${ }^{9}$

Os estudos que exploram a sazonalidade das doenças e em particular a vertigem são raros: Mariani et al ${ }^{11}$ demonstrou um aumento do número de crises de vertigem posicional paroxística benigna (VPPB) no inverno, em particular em Janeiro, e uma diminuição progressiva na primavera e no verão (mínimo em Agosto).

Deve-se alertar para a desvalorização frequente das alterações do equilíbrio como manifestação reveladora de doenças não vestibulares, como o acidente vascular cerebral e a epilepsia. ${ }^{12,13}$ Alguns estudos sugerem que o acidente vascular cerebral (AVC) poderá ser responsável por $25 \%$ das alterações do equilíbrio de apresentação aguda sem outros sinais ou sintomas neurológicos ${ }^{14}$. Outros estudos referem que o AVC é uma causa menos frequente - só $3,2 \%$ dessas apresentações. ${ }^{15} \mathrm{O}$ que interessa discutir neste trabalho é que os médicos dos SU de ORL são demonstradamente os médicos de "primeira linha" no atendimento dos doentes com alterações do equilíbrio, pelo que devem ser capazes de selecionar os casos com indicação para realizarem exames imagiológicos para identificar patologias centrais ou os casos que devem ser imediatamente referenciados para um neurologista.

Este estudo procurou obter dados sobre a epidemiologia da vertigem e do desequilíbrio num serviço de urgência de otorrinolaringologia, sem procurar identificar as suas causas específicas. No futuro, deveriam ser realizados estudos sobre a incidência e prevalência dos distúrbios vestibulares específicos nos serviços de urgência, para permitir tomar decisões clínicas ainda mais fundamentadas e meIhorar o atendimento destes doentes.

\section{CONCLUSÃO}

A vertigem num serviço de urgência apresenta características epidemiológicas específicas. Este é o primeiro trabalho que analisa estas características na população portuguesa e em particular a sua distribuição sazonal. Dos episódios de urgência na otorrinolaringologia num hospital central, $11 \%$ foram motivados por vertigem ou desequilíbrio, particularmente nas mulheres, em indivíduos idosos, e com uma distribuição sazonal, com maior expressão no verão e outono.

\section{PROTECÇÃO DE PESSOAS E ANIMAIS}

Este estudo foi analisado e aprovado pela Comissão de Ética para a Saúde (CES) do Centro Hospitalar de Lisboa Ocidental (CHLO). Os autores declaram que os procedimentos seguidos estavam de acordo com a Declaração de Helsínquia da Associação Médica Mundial.

\section{CONFIDENCIALIDADE DOS DADOS}

Os autores declaram ter seguido os protocolos do seu centro de trabalho acerca da publicação dos dados de doentes.

\section{CONFLITO DE INTERESSES}

Os autores declaram não ter nenhum conflito de interesses relativamente ao presente artigo.

\section{FONTES DE FINANCIAMENTO}

Não existiram fontes externas de financiamento para a realização deste artigo.

\section{REFERÊNCIAS}

1. Committee on Hearing and Equilibrium guidelines for the diagnosis and evaluation of therapy in Meniere's disease. American Academy of Otolaryngology-Head and Neck Foundation, Inc. Otolaryngol Head Neck Surg. 1995;113:181-5.

2. Moulin T, Sablot D, Vidry E, Belahsen F, Berger E, Lemounaud P, et al. Impact of emergency room neurologists on patient management and outcome. Eur Neurol. 2003;50:207-14.

3. Mendel B, Bergenius J, Langius-Eklof A. Dizziness: A common, troublesome symptom but often treatable. J Vestib Res. 2010;20:391-8.

4. Neuhauser HK, Radtke A, von Brevern M, Lezius F, Feldmann M, Lempert T. Burden of dizziness and vertigo in the community. Arch Intern

Med. 2008;168:2118-24.

5. Hain TC. Dizziness-and-balance.com: Epidemiology of dizziness. [consultado 2015 jan 31]; Available from: http://dizziness-and-balance. com/disorders/dizzy_epi.html.

6. Benecke H, Agus S, Kuessner D, Goodall G, Strupp M. The Burden and Impact of Vertigo: Findings from the REVERT Patient Registry. Front Neurol. 2013;4:136.

7. Burt CW, Schappert SM. Ambulatory care visits to physician offices, hospital outpatient departments, and emergency departments: United States, 1999--2000. Vital Health Stat. 2004;157:1-70.

8. Kerber KA, Meurer WJ, West BT, Fendrick AM. Dizziness presentations 
in U.S. emergency departments, 1995-2004. Acad Emerg Med. 2008;15:744-50.

9. Garrigues HP, Andres C, Arbaizar A, Cerdan C, Meneu V, Oltra JA, Santonja J, Perez A. Epidemiological aspects of vertigo in the general population of the Autonomic Region of Valencia, Spain. Acta Otolaryngol. 2008;128:43-7.

10. Lai YT, Wang TC, Chuang LJ, Chen MH, Wang PC. Epidemiology of vertigo: a National Survey. Otolaryngol Head Neck Surg. 2011;145:1106.

11. Mariani P, Pelagatti M, Hahn A, Alpini D. Epidemiology of paroxysmal positioning vertigo: correlation with seasons, climate, and pollution. Int Tinnitus J. 2008;14:168-74.

12. Ferro JM, Pinto AN, Falcao I, Rodrigues G, Ferreira J, Falcao F, Azevedo
E, Canhao P, Melo TP, Rosas MJ, Oliveira V, Salgado AV. Diagnosis of stroke by the nonneurologist. A validation study. Stroke. 1998;29:1106-9.

13. Hopkins A, Menken M, DeFriese G. A record of patient encounters in neurological practice in the United Kingdom. J Neurol Neurosurg Psychiatry. 1989;52:436-8.

14. Norrving B, Magnusson M, Holtas S. Isolated acute vertigo in the elderly; vestibular or vascular disease? Acta Neurol Scand. 1995;91:43-8.

15. Kerber KA, Brown DL, Lisabeth LD, Smith MA, Morgenstern LB. Stroke among patients with dizziness, vertigo, and imbalance in the emergency department: a population-based study. Stroke. 2006;37:2484-7.

16. Neuhauser HK, von Brevern M, Radtke A, Lezius F, Feldmann M, Ziese $\mathrm{T}$, et al. Epidemiology of vestibular vertigo: a neurotologic survey of the general population. Neurology. 2005;65:898-904. 